\title{
Overview of recent advances in molecular analysis for diagnosing early stage lung cancer nodules
}

\author{
Milo Frattini $^{1 \wedge}$, Patrizia Froesch $^{2}$, Samantha Epistolio $^{1 \wedge}$ \\ ${ }^{1}$ Laboratory of Molecular Pathology, Institute of Pathology (ICP), Cantonal Hospital (EOC), Locarno, Switzerland; ${ }^{2}$ Oncology Institute of Southern \\ Switzerland (IOSI), Cantonal Hospital (EOC), Bellinzona, Switzerland \\ Correspondence to: Dr. Milo Frattini, PhD. Laboratory of Molecular Pathology, Institute of Pathology (ICP), Cantonal Hospital - Ente Ospedaliero \\ Cantonale (EOC), Via in Selva, 24, CH-6601 Locarno, Switzerland. Email: milo.frattini@eoc.ch. \\ Comment on: Liang W, Chen Z, Li C, et al. Accurate diagnosis of pulmonary nodules using a noninvasive DNA methylation test. J Clin Invest. \\ 2021;131(10):e145973.
}

Submitted Sep 29, 2021. Accepted for publication Oct 13, 2021.

doi: $10.21037 /$ tlcr-21-802

View this article at: https://dx.doi.org/10.21037/tlcr-21-802

Lung cancer is the second most common neoplasm worldwide and the leading cause of cancer-related death (1). In 2020, this neoplastic disease accounted for $18 \%$ of all total cancer deaths, accounting for $22 \%$ of all tumor associate deaths in females and $23 \%$ in males $(1,2)$. The main reason for the high mortality rate of lung cancer is essentially related to late diagnosis. Indeed, more than $70 \%$ of cases are detected at stage II-IV, where survival is poor $(2,3)$ : the overall 5 -year survival rate for localized lung cancer is $63 \%$ and for advanced/metastatic lung cancer it drops to $7 \%$ (4).

The 5-year survival rate, for all types of lung cancer, is usually not higher than $25 \%$; for non-small lung cancers (NSCLC) it is 25\% and for small cell lung cancer (SCLC) only $7 \%$ (4). Therefore, it has become clear that there is an absolute need to identify methods for early diagnosis $(3,5,6)$.

However, to date, in daily practice, the only methodologies applied for the diagnosis of malignant pulmonary nodules (PNs) are radiological techniques and, in presence of $\mathrm{PNs}$ suspected to be malignant, the subsequent pathological evaluation of samples obtained through invasive approaches such as fine-needle biopsies, bronchoscopy or surgery $(5,6)$.

Concerning radiological techniques, it has been verified that chest radiography is of no value in the early detection of lung cancers, in contrast low-dose computed tomography (LDCT) is a good tool for detecting lung cancer at an early stage. LDCT is effective in identifying small PNs and some models have been designed based on LDCT imaging parameters (e.g., the Mayo Clinic Model and the Veterans Affairs Model) (6). The advantage of radiology is that it is minimally invasive. However, its sensitivity is largely affected by the size and location of nodules and, if repeated within a short period of time, could be hazardous to patients' health (6). In addition, although LDCT has a high sensitivity it is characterized by low specificity. Therefore, out of the many nodules classified at indeterminate risk (ranging in size between 7 and $29 \mathrm{~mm}$ ), only $1.7 \%$ to $22 \%$ are malignant (7). This uncertainty causes concern and fear in patients, who see the diagnosis as inconclusive and have to wait months for an answer or to undergo an invasive procedure such as a needle biopsy.

The second step in the investigation of suspected lung cancer lesions identified by LDCT foresees the application of the aforementioned invasive approaches. However, fineneedle biopsy or surgery present two main disadvantages. Firstly, these techniques are not always feasible due to different factors including the location of PNs and the physical conditions of the patients. Secondly, relevant and serious complications may occur after these approaches, including haemorrhage, infection and/or pneumothorax (6).

Therefore, clinical research has turned towards alternative methods, which are expected to outperform LDCT. Promising results have been obtained from the

^ ORCID: Milo Frattini, 0000-0003-2334-3412; Samantha Epistolio, 0000-0003-1236-2722. 
characterization of markers in liquid biopsies (e.g., blood, sputum, urines) because the analyses in this kind of materials are significantly less invasive, are cost-effective, can be done serially overtime without risk and have no psychological impact on the patient (8).

The latest development is represented by the results recently published by Liang and colleagues in the article entitled "Accurate diagnosis of PNs using a noninvasive DNA methylation test" (9). In this retrospective, multicenter study, they describe an alternative, costeffective and minimally invasive assay for the early diagnosis of cancerous PNs. The Authors started with a panel of 12,899 preselected lung cancer-specific methylation regions, analysed using the proprietary AnchorIRIS technology (AnchorDx, China) from which they derived a model from targeted regions sequenced on the NovaSeq 6000 System (Illumina Inc, USA) (9).

To initiate the development of the specific targeted methylation model, Liang's research group combined methylation results obtained from 232 cases with PNs (133 benign and 99 malignant) and lung cancer-specific DNA methylation markers discovered from The Cancer Genome Atlas database $(9,10)$. Afterwards, Liang and colleagues tested this preliminary model on a training set of 309 plasma cell-free DNA (cfDNA) samples in order to define the number and the specific methylated sites to be included in the final model. At the end, the definitive model, characterized by 100 methylation features and named PulmoSeek, was tested on a validation set (140 plasma samples: 40 benign and 100 malignant) achieving an Area Under the ROC (Receiver Operating Characteristic) Curve (AUC) of 0.843 and a sensitivity of 0.990 (9). The PulmoSeek model enabled the detection of lung malignancies with a sensitivity of 0.971 for stage $0-1$ and of 0.875 for later stage cancers and outperformed both the Mayo Clinic Model and the Veterans Affairs Model (AUC of 0.843 versus 0.602 and 0.512 respectively) (9). Furthermore, PulmoSeek showed better results than PETCT in identifying different types of nodules, including those that are more difficult to classify using this medical imaging technique (i.e., ground-glass nodules) (9).

The new assay seems to be very promising because, in addition to the better technical features (in terms of sensitivity and specificity), it is a more cost-effective, non-invasive approach that can also be used for the characterization of early stage lung nodules, if compared to radiology and/or tissue biopsies analyses. The strength of PulmoSeek lies in the fact that it involves the assessment of methylation and, above all, in the fact that it is carried out on plasma samples. First of all, methylation is a much more stable marker in plasma compared to other targeted analyses recently introduced in clinical practice (e.g., detection of genetic alterations in oncogenes such as point mutations, insertions, deletions and translocations; that cumulatively are present only in a fraction of cases, leading to the impossibility to be used for the identification of all cancers). The higher stability and representativeness of methylation markers in plasma, compared to the assessment of genetic alterations, is related to the fact that this epigenetic change is homozygous and that hypermethylated promoter regions are associated with repressive chromatinassociated histone modifications, in contrast to the nucleosome-free region of unmethylated and actively transcribed promoters. This provides a relative enrichment for hypermethylated promoter regions in plasma during apoptosis (11). Furthermore, hypermethylation in specific chromatin regions is a common feature among all cancer types, whereas oncogene alterations are specific to different histologic subtypes. Therefore, a test based on methylation sequences may allow detection of all types of lung cancer.

The features of PulmoSeek, in terms of high sensitivity and specificity, may further prognosticate its role in the control of patients' follow-up: instead of making serial radiological evaluations, the analysis of the methylation profile may represent a better estimate of the course of the disease with a less invasive technique.

One of the disadvantages of such an assay may be related to non-assessable cases, a number that is approximately $10 \%$ in the paper of Liang and colleagues. When it comes to DNA analysis, a non-negligible proportion of patients cannot be investigated, due to various technical problems, including poor DNA quality or those related to sample storage. It is a fact, an intrinsic feature for this type of analysis. Therefore, it is highly plausible that, apart from the fact that the turnaround time of DNA-based analyses is significantly higher than that for radiology-based technique, DNA analysis will not entirely replace the assessment by radiological technique, but these two options will both be present and will probably cooperate in the future. For example, an initial screening using radiological techniques followed by DNA analysis can be proposed as a useful algorithm.

The work of Liang and colleagues is not the first study investigating the methylation status in lung cancer patients. A very comprehensive review has recently appeared, prepared by the "father" of methylation analyses (11). 
However, the most relevant, and cited, studies, are related to a single or a few markers; for instance, the efficacy of PNs prediction models through evaluation of methylation in plasma has also been reported by Xing and colleagues (12). More importantly, all these studies are reported to be superior with respect to radiological techniques. However, although they have emerged in strong and rigorous studies, these markers appear rather extemporaneous and, in fact, none of them have entered into clinical practice. In contrast to the studies published so far, the PulmoSeek study is the first which tests a significant number of cases in both the training set and the validation set and, more importantly, assesses a large number of cases (100 in the final model) (11).

In recent years, the relevant advantages of cfDNA assessment for the early diagnosis of PNs have led to the publication of a large number of studies concerning this topic and focused on other markers that are considered more stable circulating biomarkers than methylation, such as extracellular matrix-related proteins, immunoresponse markers and microRNA (miRNA) (13-20).

Regarding the field of extracellular proteins release, the publication by Andriani and colleagues reports that soluble factors related to tumor stroma can be detected in plasma of primary lung cancer patients and may represent a valuable complementary diagnostic tool to discriminate lung cancer patients from healthy heavy-smokers individuals $(13,14)$. In particular, this study demonstrates that levels of SPARC (an extracellular matrix glycoprotein protein) in plasma, assessed by gene expression microarrays, showed good performance in discriminating the two groups ( $\mathrm{AUC}=0.744, \mathrm{P}<0.001)(13)$.

In addition to tumor stroma soluble factors, markers of immunological response in plasma could also be possible early indicators of lung cancer development (14). This topic is described in the study of Ajona et al., which illustrates how plasma levels of $\mathrm{C} 4 \mathrm{~d}$, detected by immunohistochemistry, could predict lung cancer risk in asymptomatic individuals (15). Indeed, in this study, comparison of $\mathrm{C} 4 \mathrm{~d}$ levels between a group of early stage patients and matched control subjects resulted in an $\mathrm{AUC}=0.782(\mathrm{P}<0.001)(14,15)$.

In addition to the markers described in the last paragraphs, the evaluation of miRNA in plasma could also be a good candidate to mitigate the disadvantages characterizing LDCT and invasive biopsy approaches. The relevance of miRNA analyses in plasma for the early diagnosis of lung cancer was investigated by Sozzi et al. through evaluation of prospectively collected samples from smokers in the randomized Multicenter Italian
Lung Detection (MILD) trial (16). In this work, Sozzi and co-workers identified a signature of 4 miRNAs in plasma samples enabling a correct risk stratification of subjects before and after undergoing $\operatorname{LDCT}(14,16)$. These signatures were defined by assessing the expression levels of 24 miRNAs, analyzed by real-time PCR, and the method was called miRNA Signature Classifier (MSC). More in detail, the investigators created a three-level MSC algorithm (low, intermediate, high-risk) by categorizing study participants on the basis of predefined cut-off points of positivity for four different groups, corresponding to four different miRNAs signatures: risk of disease, risk of aggressive disease, presence of disease and presence of aggressive disease (16). The miRNAs ratios of the four signatures were determined within two training sets of participating samples, independent of the validation cohort (939 cases) $(14,16)$. The prognostic performance of MSC was consequently evaluated in a second study published by the same research group and including 3,411 heavy smokers enrolled in two screening programmes based on annual or biennial LDCT and during ten years (17-19). This study demonstrated that the observed five-year survival rate was $88.9 \%$ for low risk, $79.5 \%$ for intermediate risk and $40.1 \%$ for high risk patients using the MSC algorithm $(\mathrm{P}=0.001)$ (17-19).

The importance of exosomal miRNAs is also reported in the study of Jin et al. In this article, 4 exosomal miRNAs were described as specific for lung adenocarcinoma and 3 as squamous cell lung cancer specific because they were respectively promising and effective markers of these specific subtypes of lung cancer in plasma samples (20).

Overall, as far as the analysis of methylated sequences is concerned, the reported studies, with the exclusion of Sozzi's group, are still characterized by the analysis of a low number of markers, a feature that increases the possibility of not identifying all pulmonary neoplastic lesions, thus again supporting the relevance and goodness of Liang's group study.

It can also be speculated that the marker analyses for the early identification of malignant PNs, especially methylation assessment, could be further improved in the near future. First of all, plasma analyses could be replaced by the evaluation of respiratory secretions such as salivary samples obtained from sputum, bronchoalveolar washings and pleural fluids. Evaluation of sputum samples could improve the sensitivity of methylation-based approaches compared to plasma analysis and could be easily introduced into the current screening algorithms $(11,21,22)$. Indeed, 
sputum samples are characterized by higher amounts of cfDNA produced by lung cancer cells than plasma, because respiratory secretions are closer to the primary site of this malignancy $(21,22)$.

A second improvement for PNs evaluation could be the introduction of approaches based on next-generation sequencing or digital PCR, characterized by a lower limit of detection, not only for methylation (as applied by Liang and colleagues) but also for miRNAs assessment $(11,23,24)$. The need for higher sensitivity is related to the lower amount of ctDNA in the plasma of patients with early-stage cancers compared to advanced lung cancer $(23,24)$.

The study of Liang and colleagues, as well as the other articles mentioned above, demonstrates that non-invasive biomarkers have been widely investigated because they are essential for the assessment of PNs in plasma. However, larger multicentre studies are required for clinical validation and translation of epigenetic biomarkers into the clinical diagnostic routine. Therefore, current efforts should be focused on standardizing protocols to compare different studies with the aim of large-scale collaborative studies. In particular, since multiple types of biofluids are used for the design of different assays, standardization of protocols for processing these liquid biopsies is required.

In conclusion, the study carried out by Liang's research group demonstrates how a blood-based DNA methylation model can efficiently complement or even replace radiological methodologies, such as LDCT, in the field of the early identification of malignant PNs in lung cancer.

\section{Acknowledgments}

Funding: None.

\section{Footnote}

Provenance and Peer review: This article was commissioned by the editorial office, Translational Lung Cancer Research. The article did not undergo external peer review.

Conflicts of Interest: All authors have completed the ICMJE uniform disclosure form (available at https://dx.doi. org/10.21037/tlcr-21-802). Dr. PF reports having an advisory role for Roche, Pfizer, Boehringer Ingelheim, Takeda, MSD, BMS. The other authors have no conflicts of interest to declare.

Ethical Statement: The authors are accountable for all aspects of the work in ensuring that questions related to the accuracy or integrity of any part of the work are appropriately investigated and resolved.

Open Access Statement: This is an Open Access article distributed in accordance with the Creative Commons Attribution-NonCommercial-NoDerivs 4.0 International License (CC BY-NC-ND 4.0), which permits the noncommercial replication and distribution of the article with the strict proviso that no changes or edits are made and the original work is properly cited (including links to both the formal publication through the relevant DOI and the license). See: https://creativecommons.org/licenses/by-nc-nd/4.0/.

\section{References}

1. Siegel RL, Miller KD, Jemal A. Cancer statistics, 2020. CA Cancer J Clin 2020;70:7-30.

2. Sung H, Ferlay J, Siegel RL, et al. Global Cancer Statistics 2020: GLOBOCAN Estimates of Incidence and Mortality Worldwide for 36 Cancers in 185 Countries. CA Cancer J Clin 2021;71:209-49.

3. Andriani F, Conte D, Mastrangelo T, et al. Detecting lung cancer in plasma with the use of multiple genetic markers. Int J Cancer 2004;108:91-6.

4. Lung Cancer - Non-Small Cell: Statistics. Accessed /08/23/2021. Available online: https://www.cancer.net/ cancer-types/lung-cancer-non-small-cell/statistics

5. McWilliams A, Tammemagi MC, Mayo JR, et al. Probability of cancer in pulmonary nodules detected on first screening CT. N Engl J Med 2013;369:910-9.

6. Nair VS, Sundaram V, Desai M, et al. Accuracy of Models to Identify Lung Nodule Cancer Risk in the National Lung Screening Trial. Am J Respir Crit Care Med 2018;197:1220-3.

7. Gierada DS, Pinsky P, Nath H, et al. Projected outcomes using different nodule sizes to define a positive CT lung cancer screening examination. J Natl Cancer Inst

8. Strotman LN, Millner LM, Valdes R Jr, et al. Liquid Biopsies in Oncology and the Current Regulatory Landscape. Mol Diagn Ther 2016;20:429-36.

9. Liang W, Chen Z, Li C, et al. Accurate diagnosis of pulmonary nodules using a noninvasive DNA methylation test. J Clin Invest 2021;131:145973.

10. Chang JT, Lee YM, Huang RS. The impact of the Cancer Genome Atlas on lung cancer. Transl Res 2015;166:568-85.

11. Farooq M, Herman JG. Noninvasive Diagnostics for Early Detection of Lung Cancer: Challenges and Potential 
with a Focus on Changes in DNA Methylation. Cancer Epidemiol Biomarkers Prev 2020;29:2416-22.

12. Xing W, Sun H, Yan C, et al. A prediction model based on DNA methylation biomarkers and radiological characteristics for identifying malignant from benign pulmonary nodules. BMC Cancer 2021;21:263.

13. Andriani F, Landoni E, Mensah M, et al. Diagnostic role of circulating extracellular matrix-related proteins in nonsmall cell lung cancer. BMC Cancer 2018;18:899.

14. Sozzi G, Boeri M. Potential biomarkers for lung cancer screening. Transl Lung Cancer Res 2014;3:139-48.

15. Ajona D, Pajares MJ, Corrales L, et al. Investigation of complement activation product $\mathrm{c} 4 \mathrm{~d}$ as a diagnostic and prognostic biomarker for lung cancer. J Natl Cancer Inst 2013;105:1385-93.

16. Sozzi G, Boeri M, Rossi M, et al. Clinical utility of a plasma-based miRNA signature classifier within computed tomography lung cancer screening: a correlative MILD trial study. J Clin Oncol 2014;32:768-73.

17. Sestini S, Boeri M, Marchiano A, et al. Circulating microRNA signature as liquid-biopsy to monitor lung cancer in low-dose computed tomography screening. Oncotarget 2015;6:32868-77.

18. Mensah M, Borzi C, Verri C, et al. MicroRNA Based Liquid Biopsy: The Experience of the Plasma miRNA

Cite this article as: Frattini $M$, Froesch $\mathrm{P}$, Epistolio S. Overview of recent advances in molecular analysis for diagnosing early stage lung cancer nodules. Transl Lung Cancer Res 2021;10(11):4303-4307. doi: 10.21037/tlcr-21-802
Signature Classifier (MSC) for Lung Cancer Screening. J Vis Exp 2017.

19. Fortunato O, Gasparini P, Boeri M, et al. Exo-miRNAs as a New Tool for Liquid Biopsy in Lung Cancer. Cancers (Basel) 2019;11:888.

20. Jin X, Chen Y, Chen H, et al. Evaluation of TumorDerived Exosomal miRNA as Potential Diagnostic Biomarkers for Early-Stage Non-Small Cell Lung Cancer Using Next-Generation Sequencing. Clin Cancer Res 2017;23:5311-9.

21. Belinsky SA, Liechty KC, Gentry FD, et al. Promoter hypermethylation of multiple genes in sputum precedes lung cancer incidence in a high-risk cohort. Cancer Res 2006;66:3338-44.

22. Hulbert A, Jusue-Torres I, Stark A, et al. Early Detection of Lung Cancer Using DNA Promoter Hypermethylation in Plasma and Sputum. Clin Cancer Res 2017;23:1998-2005.

23. Bailey VJ, Zhang Y, Keeley BP, et al. Single-tube analysis of DNA methylation with silica superparamagnetic beads. Clin Chem 2010;56:1022-5.

24. Liu B, Chi W, Li X, et al. Evolving the pulmonary nodules diagnosis from classical approaches to deep learning-aided decision support: three decades' development course and future prospect. J Cancer Res Clin Oncol 2020;146:153-85. 